\title{
Modelado para la determinación del poder calorífico de la gasificación de la balsa en instalaciones downdraft mediante redes neuronales artificiales*
}

\section{Eddy Bladimir Ibarra-Sánchez Álvaro Luis Oñate-Chiliquinga Ángelo Homero Villavicencio-Poveda}

\author{
Universidad de las Fuerzas Armadas "Espe" \\ Departamento de Ciencias de la Energía y Mecánica \\ CP 171103 \\ ECUADOR
}

\section{correos electrónicos (emails): \\ ebibarra@espe.edu.ec, alonate@espe.edu.ec ergutierrez@espe.edu.ec, ahvillavicencio@espe.edu.ec}

Recibido 13-02-2020, aceptado 29-04-2020.

\section{Resumen}

En este trabajo se presentan los principales resultados investigativos obtenidos por los autores en el modelado para la predicción del poder calorífico del gas de síntesis obtenido en instalaciones de gasificación termoquímica downdraft de la madera de balsa, con la incorporación de técnicas basadas en redes neuronales artificiales. Se realizó un análisis del estudio del estado del arte de trabajos de investigación previos vinculados al modelado matemático de estas instalaciones por las diferentes técnicas reflejada en la literatura especializada. El modelado se lleva a cabo mediante una planificación experimental $3^{\mathrm{n}}$, con lo que se obtuvo los datos experimentales a los cuales se aplican técnicas de predicción mediante redes neuronales con ayuda de Matlab con resultados satisfactorios. La selección de variables para realizar la experimentación toma en cuenta la ubicación geográfica de donde se obtiene el residuo forestal de la balsa, ya que esta se produce en un clima tropical cálido-húmedo.

Por literatura se conoce que uno de los factores que influye notablemente en el poder calorífico es la humedad. Obviamente la cantidad oxígeno contenida en el aire en el proceso es regulada por una válvula de admisión, además de que es un factor preponderante la masa añadida al proceso. Teniendo en cuenta esto, la red neuronal artificial obtenida permite la predicción del poder calorífico resultante de la gasificación de la balsa con un error de $\pm 2.6 \mathrm{MJ} / \mathrm{g}$ y un ajuste del $86 \%$, lo cual permite realizar una predicción adecuada.

Palabras clave: operación de instalaciones energéticas, gasificación, biomasa, modelado matemático, redes neuronales artificiales, poder calorífico.

\section{Abstract \\ (Determination of the Calorific Power Value of the Balsa Wood through Artificial Neural Networks Modeling in a Downdraft Gasification Facility)}

This work presents the main research results obtained by the authors in the model based on the prediction the calorific power of the synthesis gas obtained through thermochemical downdraft gasification facilities of the boat wood with the of techniques of artificial neural networks. An analysis of the state-of-the-art study of previous research work linked to the mathematical modeling of these facilities was made out by the different techniques reflected in the specialized literature. The modeling is carried out using experimental factor $3^{\mathrm{n}}$, which is applied in the adquisition of experimental data; with the help of Matlab the prediction tecniques applied on neural networks are performed over the data with satisfactory results. The selection of variables for experimentation takes into account the geographical location from which the forest waste is obtained from the raft, as it is produced in a warm- humid tropical climate.

Literature is known that one of the factors that significantly influences calorific power is moisture. Obviously, the amount of oxygen contained in the air in the process is regulated by an intake valve, furthermore is a predominant factor in the mass added to the process. Considering this, the artificial neural network obtained allows the prediction calorific power resulting from the gasification of the raft with an error of $2.6 \mathrm{MJ} / \mathrm{g}$ and an adjustment of $86 \%$, which allows for an appropriate prediction.

Index terms: gasification, biomass, mathematical modeling, artificial neural networks, caloric power. 


\section{Introducción}

El creciente consumo energético de nuestra sociedad, unido a la persistencia de la crisis energética global por el agotamiento de las fuentes energéticas tradicionales, ha propiciado la búsqueda de fuentes alternativas de energía que permitan aminorar la utilización de estas reservas energética no renovables. Una de las fuentes mayoritarias presentes está constituida por la biomasa, de la que se dispone en abundancia y tiene mucho menor impacto al medio ambiente que los combustibles tradicionales [1].

La gasificación de la biomasa es un proceso complejo que involucra varias reacciones químicas y procesos de intercambio de calor y masa. La gasificación es la conversión de un caudal de materia sólida en un combustible gaseoso o líquido que puede ser quemado para liberar energía o ser utilizado para la producción de sustancias químicas valiosas [2]. Este proceso requiere de un agente gasificante (aire, oxígeno o vapor) para reordenar la estructura molecular de la biomasa y convertirla en un combustible útil con el incremento de la relación hidrógeno-carbón $(\mathrm{H} / \mathrm{C})$ [3].

En general, el proceso de gasificación depende de varios factores, entre ellos se destaca la temperatura de pirólisis, parámetro que determina el rompimiento de la molécula de la biomasa mediante la reacción de reducción que se produce a una presión determinada dentro del reactor [4]. El análisis real del proceso de gasificación, por su complejidad y diversidad de procesos termoquímicos presenta numerosas dificultades de análisis, razón por la cual los modelos matemáticos tienen vital importancia en la predicción del comportamiento del reactor, ya que proveen sistemas de ecuaciones que permiten simular o predecir el comportamiento de la instalación energética [5], [6], [7], [8], [9], [10], [16]. [21], [22], en estas dos últimas investigaciones se estudian biomasas residuales. En [21], la biomasa de residuos agrícolas de cacao, Cacao CCN51. Mientras que en [22], se estudian los residuos de Eichhorniacrassipes "lechugín", cascarilla de arroz y leña. Además, en un reciente estudio, [23] elaboraron modelos matemáticos para la estimación de los poderes calóricos superior e inferior, velocidad de ignición superior e inferior y densidad obtenida como función de los parámetros de fabricación para lograr una combustión completa en el olote de Zea Mays $L$.

En la bibliografía especializada se reflejan algunos trabajos sobre diferentes tipos de modelado de este proceso. [5] Utilizan modelos de equilibrio termodinámico para predecir la composición del gas de síntesis al suponer una mezcla de reactivos en un tiempo infinito. La dificultad de empleo de este método radica en la posible introducción de errores por la no consideración de todas las reacciones existentes en el proceso. [2] Emplea un modelo no estequiométrico en el que se minimiza la energía libre de Gibbs, el cual, aunque no se toma en cuenta la presencia de las reacciones, su análisis y correcto planteamiento es más complejo por lo que se tiende a realizar simplificaciones en la determinación de los parámetros termodinámicos, con lo que se reduce el grado de generalización [11].

En [12] se emplea un modelo de tipo cinético que, al igual que el modelo anterior, se enfoca en realizar un análisis al gas de síntesis producto del proceso y predecir su rendimiento en función de su composición en un volumen de control para un tiempo dado. Con este modelo se predicen los perfiles tanto de composición del syngas como de temperatura dentro del reactor. Aquí se considera la cinética existente en las diferentes reacciones, las condiciones hidrodinámicas y balances de masa y energía. A pesar ello, estos modelos requieren de la determinación de parámetros hidrodinámicos que suelen ser difíciles de obtener, además de que requieren un gran consumo de recursos computacionales para su resolución [13], [15]. Como se puede notar, el modelado a partir de estos tipos de modelos resulta ser muy complejo por la necesidad de incorporar en los procedimientos de cálculo parámetros termodinámicos y cinéticos que son difíciles de precisar y de los que, en la mayoría de los casos, se carece de información precisa por lo cual se requiere de realización previa de mediciones experimentales. Por estas razones, algunos autores han empleado técnicas de modelado avanzadas que permiten la predicción del comportamiento de los sistemas a partir de la adquisición de datos menos complejos de obtener experimentalmente.

En este sentido, [14] y [17] reflejan, en su revisión y análisis de los modelos de gasificación de biomasa, el empleo de las redes neuronales para predecir el comportamiento de un reactor para producción del gas y otros componentes, Estos autores realizan una descripción del proceso de creación de un modelo de red neuronal artificial que sirve como estimador del proceso de gasificación medido en parámetros no difíciles de modelar y demuestran la capacidad de aproximación de este tipo de modelos, incluso al considerar otros intervalos de valores de las variables empleadas para la predicción de indicadores.

Por las razones expuestas anteriormente, el alcance que presenta este artículo es el desarrollo de un modelo de predicción del poder calorífico del gas resultante de la gasificación de la madera en un reactor de tipo downdraft mediante redes neuronales artificiales y la realización de mediciones experimentales activas.

\section{Materiales y métodos}

En la determinación del poder calorífico del gas obtenido de un proceso de gasificación es necesario la consideración no solo de los parámetros termodinámicos, también es necesario 
considerar aquellos parámetros de operación del gasificador que permiten variar las condiciones de realización de este proceso. Básicamente, la regulación de las condiciones internas del reactor se determina en función de la temperatura de pirolización, la cantidad de biomasa que se introduce y el caudal de aire que se incorpora al sistema.

Este último parámetro de operación se regula a partir de la apertura de una válvula que posibilita la succión del aire para que se produzca el efecto cigarro requerido para la generación de gas. Esta entrada de aire también regula el caudal de aire de combustión que entra al gasificador y por tanto también permite regular la temperatura en su interior. La humedad de la biomasa también constituye un parámetro crucial en la calidad del proceso de gasificación pues su presencia consume energía como parte del proceso previo de secado además de aportar vapor de agua como parte de los gases que se generan.

A partir de este análisis, las variables independientes por considerar para la predicción del poder calorífico del gas son:

- Humedad, Hu.

- Cantidad de biomasa, Cbio.

- Porciento de apertura de la válvula, $A p V$.

Para estas variables se seleccionaron los niveles: $12 \%, 16 \%$, $20 \%$; $1 \mathrm{~kg}, 3 \mathrm{~kg}, 5 \mathrm{~kg} ; 25 \%$, 50\% y $100 \%$, respectivamente. Como variable dependiente se define:

- El poder calórico del gas, $P C$.

El plan experimental se concibió con una estructura $3 n$ (niveles alto, medio y bajo, para tres variables, $n=3$ ) con tres réplicas para un total de 81 juegos de datos. Esta estructura de experimentación tiene como finalidad la consideración del carácter no lineal de las relaciones entre las variables y para reflejar los factores casuales que se presenten, incluyendo los errores de medición.

La instalación experimental constituida por un gasificador tipo downdraft con una capacidad de $10 \mathrm{~kW}$. El aseguramiento experimental está compuesto por un medidor de humedad MD914 con un intervalo de medición de 2 a $60 \%$ con una resolución de $0.5 \%$, un sensor de nivel controlado mediante una paleta para censar el nivel de biomasa, una alarma digital de detección de $\mathrm{CO}$ marca Kidde con un intervalo de medición de 30-999 PPM con $\pm 30 \%$ de error. Las mediciones del poder calorífico del gas generado se realizaron con un cromatógrafo de gases con espectrómetro de masa GC-MS a partir de muestras almacenadas en bolsas colectoras de plástico esterilizadas y extraídas del colector de gas. El control se lo realiza en un programa desarrollado en el software LabVIEW de la National Instruments.

El proceso de control del gasificador empieza con la medición en su gran mayoría de las termocuplas cuyas señales son enviadas a una tarjeta de adquisición de datos myRIO quien al mismo tiempo envía la señal transformada a un computador, donde se puede verificar al mismo tiempo la entrada de aire por medio de la apertura o cierre en forma manual de una válvula de admisión de aire conectada a un sistema Venturi, el mismo que absorberá el aire en el reactor y al mismo tiempo el gas producido para obtener el efecto de gasificación, ya que este proceso se produce por una baja cantidad de oxígeno.

El objetivo de esta experimentación es proporcionar los datos numéricos necesarios para la predicción de los valores de poder calorífico del gas generado por el gasificador como función de los parámetros de entrada indicados y como se muestra en la expresión (1). Estos parámetros han sido considerados representativos del sistema para poder evaluar el poder calorífico inferior y superior de una biomasa como lo mencionan [21], [22] en sus respectivas investigaciones.

$$
P C=f(H u, C b i o, A p V)
$$

Debido al poco grado de relación analítica que se presentan entre las variables independientes y la variable dependiente, es necesario la utilización de una estructura de modelado que permita determinar, bajo ciertas reglas funcionales, los valores de salida del modelo según los valores de las variables indicadas como entradas [19]. Para ello, se utilizan las técnicas de inteligencia artificial, dentro de las que se destacan las redes neuronales artificiales para la predicción de datos [18], [20].

\section{Selección del algoritmo de regularización bayesiana}

La regularización bayesiana es una de las más poderosas técnicas de entrenamiento de perceptores multicapa. Tiene una alta capacidad de generalizar el ajuste de la red con un bajo sobre entrenamiento de la red mediante la optimización de los pesos de la red neuronal simultáneamente con el análisis de la capacidad de generalización de la red. Este sobreentrenamiento significa que la red ha aprendido la información que se le ha suministrado en el proceso de aprendizaje a tal punto que ha modelado el ruido presente en la misma. La selección del algoritmo para la elaboración de la red neuronal se realizó a partir de múltiples entrenamientos seleccionando grupos aleatorios de datos de entrada y de salida y comparando los resultados finales (véase Tabla 3), fundamentalmente la capacidad de ajuste de la red a los datos suministrados para el entrenamiento y validación (70\% vs $30 \%$ ), siendo el algoritmo de regularización bayesiana el de mejores resultados durante las pruebas realizadas. 


\section{Diseño experimental}

El plan experimental se concibió con una estructura $3^{\mathrm{N}}$ (alto, medio y bajo, por variables) con tres réplicas, con el fin de tomar en consideración el carácter necesariamente no lineal de las relaciones requeridas y que queden reflejados los factores casuales, incluyendo los errores de medición, para modelar los indicadores de desempeño como función de las variables de operación, con ayuda de modelos de regresión no lineales. Para la recopilación automatizada de la información experimental, se diseñó e implementó en la instalación una tarjeta de adquisición de datos myRIO y la interface maquina hombre con un programa desarrollado en LabView.

La instrumentación utilizada consta de una alarma digital de detección de $\mathrm{CO}$ marca Kidde con una intervalo de medición de 30-999 PPM con error $\pm 30 \%$ de la magnitud medida en condiciones normales, un medidor de humedad MD914 con un intervalo de medición de 2 a $60 \%$ una resolución de $0.5 \%$ un sensor de nivel de combustible controlado mediante una paleta para censar el nivel de biomasa, 8 termocuplas tipo $k$, con intervalo de medición de 0 a $1250^{\circ} \mathrm{C}$ de temperatura con un límite de error $\pm 2.2 \%{ }^{\circ} \mathrm{C}$ de chromel -alumel y un cable de compensación $\mathrm{KX}$ - ubicadas de la siguiente forma: cuatro para las zonas de reducción, pirolisis, combustión y secado, una en la descarga de cenizas, y tres para censar las temperaturas del gas a la entrada del ciclón, a la entrada del intercambiador de calor y a la salida del intercambiador de calor, así como de dos manómetros de agua tipo $U$ con intervalo de medición de $200 \mathrm{~mm} \mathrm{de} \mathrm{H}_{2} \mathrm{O}$, con error de medición de $\pm 1 \mathrm{~mm} \mathrm{de} \mathrm{H}_{2} \mathrm{O}$ uno de ellos toma la diferencia de presión existente en el reactor y el otro en la placa orificio para medición de caudal másico de aire de ingreso en las diferentes aperturas de válvula. La disposición de toda la instrumentación se ilustra en la Fig. 1.

Para elaborar las ecuaciones de regresión que se ajusten adecuadamente a los resultados experimentales se utilizan las técnicas de linealización de modelos expuesta en el libro Sistemas de Ingeniería [16].

En particular, si a ambos miembros del modelo monomial (1), como se puede observar en la ecuación 2 :

$$
y=c x_{1}^{\alpha_{1}} x_{2}^{\alpha_{2}} \ldots x_{n}^{\alpha_{n}}
$$

donde

$$
c, \alpha_{1}, \alpha_{2}, \ldots, \alpha_{n} \in R \neq 0
$$

Se le hallan los logaritmos que se obtiene de la ecuación 3:

$$
\log y=\log c+\alpha_{1} \log x_{1}+\alpha_{2} \log x_{2}+\ldots+\alpha_{n} \log x
$$

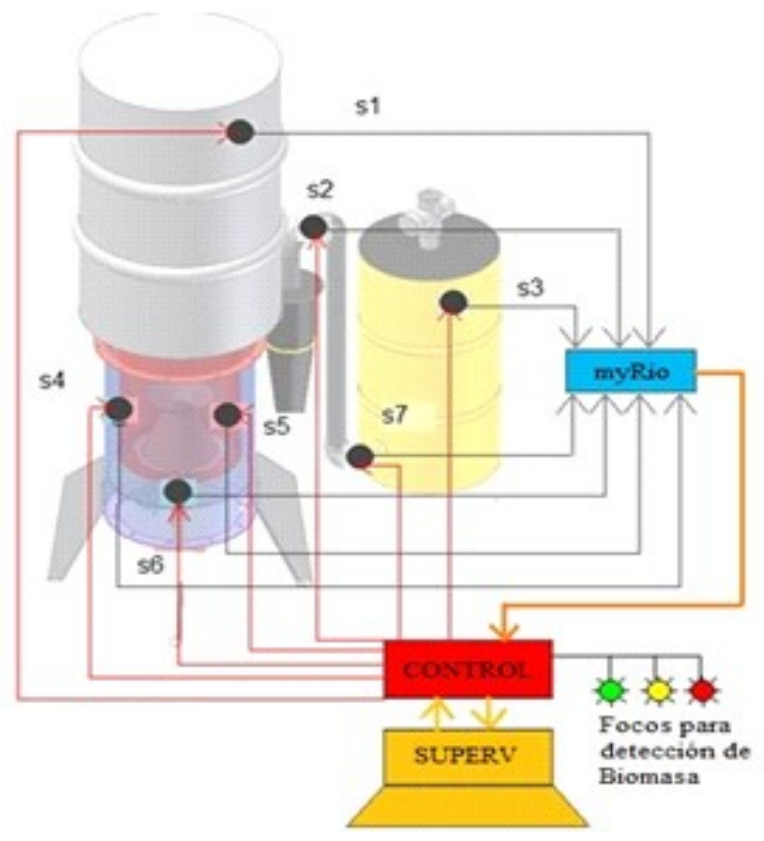

Fig. 1. Puntos de control del gasificador tipo downdraft. s1: termocupla 1 (temperatura ambiente), s2: termocupla 2 (temperatura del gas a la salida del ciclón), s3: termocupla 3 (temperatura del gas a entrada a la TEA de combustión), s4: termocupla 4 (temperatura en la zona de pirolisis), s5: termocupla 5 (temperatura en la zona de combustión), s6: termocupla 6 (temperatura de cenizas), s7: termocupla 7 (temperatura de entrada al filtro).

de donde se deduce que los coeficientes $\alpha_{1}, \alpha_{2}, \ldots, \alpha_{n}$ constituyen potencias de mejor ajuste del monomial (2) o de cualquier composición de productos $x_{i}^{\alpha i} x_{j}^{\alpha j} \ldots$.

De igual forma se pueden generar términos exponenciales, recíprocos y otros. Se utilizará el siguiente esquema de búsqueda de modelos [16]:

1. Como primer paso se selecciona todos los factores considerados "sospechosos" que tengan influencia directa en la variable dependiente y generación de los factores analizados. En este punto se utilizaron algunos procedimientos considerados como auxiliares diferentes. Por ejemplo, los factores de interacción entre las variables se pueden establecer mediante un modelo monomial auxiliar, que incluye todas las variables de la interacción; luego de hallada la ecuación de regresión de este modelo auxiliar queda esclarecidos los exponentes más adecuados del factor de interacción.

2. Como resultado se obtiene una expresión matemática de regresión donde intervienen todos los factores considerados como "sospechosos" ( $k$ factores). 
3 . Se procede a registrar un coeficiente de correlación múltiple, además el error estándar de la expresión matemática (ecuación).

4. Se encuentra una ecuación nueva con regresión de $k-1$ factores, excluyendo el factor que tiene el coeficiente más pequeño de la correlación parcial.

5. Al observar el incremento del coeficiente de correlación múltiple o el error estándar, es necesario repetir el proceso (al inicio los dos coeficientes tienden a mejorar, sin embargo, en los posterior el coeficiente de correlación múltiple empezará a disminuir mientras que el error estándar continuará con tendencia a bajar).

6. Como punto final, es importante verificar que se cumplan todos los puntos anteriores. Para el intervalo de un error estándar $2 \sigma \leq \delta$, $(\sigma$ representa el error estándar de la ecuación y $\delta$ es el error máximo).

\section{Resultados}

Para crear la red neuronal artificial (RNA) se utilizó la herramienta denominada Neural Net Fitting perteneciente al Deep Learning Toolbox 12.0 de Matlab R2018b (licencia estudiantil), la que permite crear RNA para la resolución de problemas de ajustes utilizando redes de tipo feed-forward con hasta dos capas de neuronas ocultas. Esta herramienta realiza, además, toda la gestión de información de entrada-salida para poder variar operativamente la configuración de la red y visualizar el comportamiento de los indicadores $R^{2}$ y el error de ajuste mediante el MSE con respecto a los datos seleccionados para entrenar la red y para su validación (véase tablas $1,2$ y 3$)$.

Para el entrenamiento de las redes se adoptó un $15 \%$ de los datos para la validación, un 15\% para la verificación y el $70 \%$ para el entrenamiento. En la capa oculta se utilizan seis neuronas, según se puede observar en la Fig. 2. Este entrena-

Tabla 1. Resumen de resultados de las muestras obtenidas según el perfil de secado.

\begin{tabular}{|c|c|c|c|c|c|c|}
\hline \multicolumn{2}{|c|}{ Perfil secado rápido Pr_02 } & & & & & \\
\hline Temp. Trabajo $70^{\circ} \mathrm{C}$ & Masa Inicial $[\mathrm{Mi}][\mathrm{g}]$ & \multicolumn{5}{|c|}{$1,5027[\mathrm{~g}]$} \\
\hline \multirow{4}{*}{$\square^{\mathrm{t}} \mathrm{tmp} \quad 120 \mathrm{c}$} & Tiempo [min] & 1 & 2 & 3 & 4 & 5 \\
\hline & Pérdida de masa porcentual $[\% \mathrm{M}]$ & 6.908 & 7.7316 & 8.0438 & 8.1235 & 8.1966 \\
\hline & Masa seca conseguida \%D & 93.0521 & 92.2684 & 91.9495 & 91.8765 & 91.8034 \\
\hline & $\% \mathrm{R}$ & 7.505 & 8.3775 & 8.7553 & 8.889 & 8.9284 \\
\hline Figura 1. Esquema de Comportamiento del Perfil Rápido & Masa Final a los $\mathrm{N} \min [\mathrm{g}]$ & 1.4019 & 1.3891 & 1.3845 & 1.3833 & 1.3821 \\
\hline \multicolumn{7}{|c|}{ Perfil secado suave Pr_03 } \\
\hline Temp. Trabajo $70^{\circ} \mathrm{C}$ & Masa Inicial $[\mathrm{Mi}][\mathrm{g}]$ & \multicolumn{5}{|c|}{$1,5000[\mathrm{~g}]$} \\
\hline \multirow{4}{*}{$\overbrace{}^{\mathrm{tmP}} \quad 120 \mathrm{c}$} & Tiempo [min] & 1 & 2 & 3 & 4 & 5 \\
\hline & Pérdida de masa porcentual $[\% \mathrm{M}]$ & 1.3332 & 3.0998 & 4.7797 & 6.2263 & 7.2662 \\
\hline & Masa seca conseguida $\% \mathrm{D}$ & 98.6268 & 96.8602 & 95.187 & 93.7471 & 92.7072 \\
\hline & $\% \mathrm{R}$ & 1.4129 & 3.2771 & 5.1005 & 6.7003 & 7.8743 \\
\hline Figura 2. Esquema de Comportamiento del Perfil Suave & Masa Final a los $\mathrm{N} \min [\mathrm{g}]$ & 1.4806 & 1.4542 & 1.4288 & 1.407 & 1.3914 \\
\hline \multicolumn{7}{|c|}{ Perfil secado escalonado Pr_04 } \\
\hline \multirow[t]{2}{*}{ Temp. Trabajo $70^{\circ} \mathrm{C}$} & Masa Inicial $[\mathrm{Mi}][\mathrm{g}]$ & \multicolumn{5}{|c|}{$1,4998[\mathrm{~g}]$} \\
\hline & Tiempo [min] & 1 & 2 & 3 & 4 & 5 \\
\hline \multirow[t]{3}{*}{$\overbrace{{ }_{t m p 2}^{t m p}}^{120} \mathrm{c}$} & Pérdida de masa porcentual $[\% \mathrm{M}]$ & 3.5767 & 4.5776 & 5.2983 & 5.7387 & 5.9722 \\
\hline & Masa seca conseguida $\% \mathrm{D}$ & 96.3699 & 95.409 & 94.695 & 94.2747 & 94.0278 \\
\hline & $\% \mathrm{R}$ & 3.7955 & 4.8339 & 5.6021 & 6.0881 & 6.3516 \\
\hline Figura 3. Esquema de Comportamiento del Perfil Escalonado & Masa Final a los $\mathrm{N} \min [\mathrm{g}]$ & 1.4452 & 1.4303 & 1.4194 & 1.4128 & 1.4091 \\
\hline
\end{tabular}


Tabla 2. Parámetros para la toma de datos del poder calorífico de la madera de balsa.

ÁCIDO BENZOICO

\begin{tabular}{|c|c|c|c|}
\hline 1 & Valor calorífico del ácido benzoico & $\mathrm{J} / \mathrm{gr}$ & 26454 \\
\hline 2 & Peso del ácido benzoico quemado & $\mathrm{gr}$ & 1 \\
\hline 3 & Energía liberada por el ácido & $\mathrm{J}$ & 26454 \\
\hline 4 & Temperatura inicial & ${ }^{\circ} \mathrm{C}$ & 21 \\
\hline 5 & Temperatura final & ${ }^{\circ} \mathrm{C}$ & 23,29 \\
\hline 6 & Elevación de temperatura & ${ }^{\circ} \mathrm{C}$ & 2,29 \\
\hline 7 & Capacidad calorífica total & $\mathrm{kJ} /{ }^{\circ} \mathrm{C}$ & 11,551 \\
\hline
\end{tabular}

miento está basado en estudios anteriores [24]. En la Fig. 3 se indica la estructura de la red neuronal finalmente obtenida

La función de trasferencia empleada para la realización del ajuste es la función tangente sigmoidal, la cual es establecida por defecto por esta herramienta de Matlab. Esta función entrega una salida que se encuentra en el rango de (-1.0 a 1.0) y es utilizada con frecuencia para el ajuste de datos en problemas similares al estudiado [20]. El entrenamiento de la red se realizó mediante el algoritmo de regularización bayesiana, el cual, si bien requiere más tiempo de cálculo para la determinación de los coeficientes de pesos w en los nodos de la red, por la utilización de un procedimiento de tipo adaptativo, resultó ser el más adecuado para el ajuste de los datos según las pruebas realizadas a priori con todas las opciones de algoritmos disponibles a emplear.

A partir de los entrenamientos realizados para cada tipo de gasolina, se determinaron los coeficientes óptimos de pesos $w$ y los coeficientes bias $b$ para la capa oculta $\left(w_{c o}\right.$ y $\left.b_{c o}\right)$ y para la capa de salida $\left(w_{c s} \mathrm{y} b_{c s}\right)$ en dada red elaborada. En lo adelante se muestran los valores correspondientes.

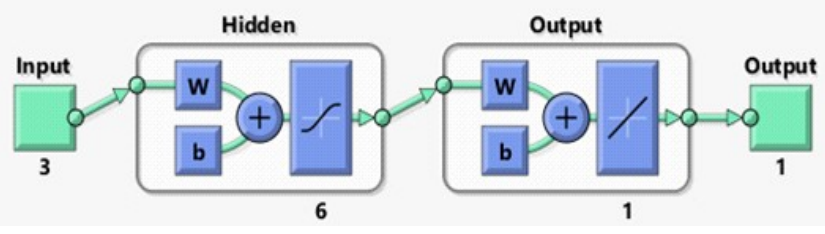

Fig. 2. Esquema general de la RNA empleada. Figura generada por Matlab R2018b.

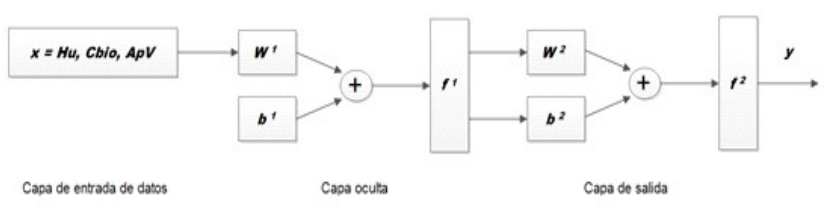

Fig. 3. Estructura de la red neuronal encontrada para todas y cada una de las salidas.

$$
b_{c o}=\left[\begin{array}{l}
-0.3688 \\
0.1408 \\
-1.9988 \\
1.78437 \\
-0.7797 \\
-0.34859
\end{array}\right]
$$

$$
w_{c o}=\left[\begin{array}{lll}
0.7528 & -0.1012 & -0.7479 \\
-0.1219 & 0.7359 & 0.1844 \\
0.8664 & -0.3137 & -1.1662 \\
-1.0887 & 1.1292 & 1.2528 \\
-0.5401 & 0.7283 & 0.5136 \\
-1.1680 & 0.1279 & 0.7265
\end{array}\right]
$$

Tabla 3. Muestras representativas utilizadas en el entrenamiento de las redes neuronales artificiales.

\begin{tabular}{|c|c|c|c|c|c|c|c|c|c|c|c|}
\hline \multicolumn{2}{|c|}{ MUESTRA } & & $\begin{array}{c}\text { Muestra } \\
1\end{array}$ & $\begin{array}{c}\text { Muestra } \\
2\end{array}$ & $\begin{array}{c}\text { Muestra } \\
3\end{array}$ & $\begin{array}{c}\text { Muestra } \\
4\end{array}$ & $\begin{array}{c}\text { Muestra } \\
5\end{array}$ & $\begin{array}{c}\text { Muestra } \\
6\end{array}$ & $\begin{array}{c}\text { Muestra } \\
7 \\
\text { (Fresca) }\end{array}$ & $\begin{array}{c}\text { Muestra } \\
8\end{array}$ & $\begin{array}{c}\text { Muestra } \\
9\end{array}$ \\
\hline 1 & Peso del combustible & $\mathrm{gr}$ & 0,8 & 0,8 & 0,8 & 0,8 & 0,8 & 0,8 & 0,8 & 0,8 & 0,8 \\
\hline 2 & Temperatura inicial & ${ }^{\circ} \mathrm{C}$ & 21 & 21 & 21 & 21 & 21 & 21 & 21 & 21 & 21 \\
\hline 3 & Temperatura final & ${ }^{\circ} \mathrm{C}$ & 22,32 & 22,34 & 22,34 & 22,36 & 22,37 & 22,24 & 22,3 & 22,37 & 22,28 \\
\hline 4 & Elevación de temperatura & ${ }^{\circ} \mathrm{C}$ & 1,32 & 1,34 & 1,34 & 1,36 & 1,37 & 1,24 & 1,3 & 1,37 & 1,28 \\
\hline 5 & Energía total liberada & $\mathrm{kJ}$ & 15,248 & 15,479 & 15,479 & 15,710 & 15,826 & 14,324 & 15,017 & 15,826 & 14,786 \\
\hline 6 & $\begin{array}{c}\text { Valor calorífico de la } \\
\text { muestra }\end{array}$ & $\mathrm{MJ} / \mathrm{kg}$ & 19,060 & 19,349 & 19,349 & 19,638 & 19,782 & 17,905 & 18,771 & 19,782 & 18,483 \\
\hline 7 & $\begin{array}{c}\text { Presión de Oxígeno } \\
\text { utilizada }\end{array}$ & $\mathrm{Bar}$ & 15 & 15 & 18 & 20 & 20 & 20 & 20 & 15 & 22 \\
\hline
\end{tabular}




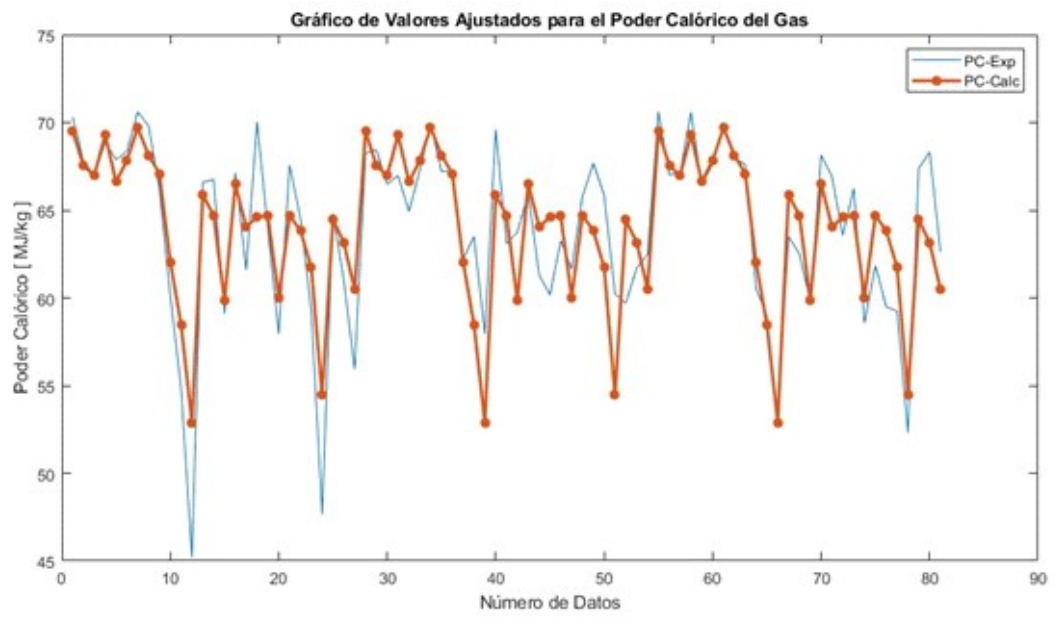

Fig. 4. Ajuste de la RNA desarrollada para la predicción del poder calorífico del gas.

$b_{s}=[1.3697]$

$$
w_{c s}=\left[\begin{array}{llllll}
-0.7098 & -0.7504 & 17938 & 1.2453 & 0.8835 & -0.6736
\end{array}\right]
$$

El porcentaje de ajuste obtenido mediante la predicción de la red neuronal artificial es de $R^{2}=86.07 \%$ con un error $S=2.63$. Para analizar la bondad de ajuste de la RNA elaborada se genera el gráfico de ajustes de los valores estimados y los valores predichos para el 100\% de los datos de entrada. En la Fig. 2 se muestran el gráfico correspondiente a cada uno de los valores de poder calorífico de la biomasa predicho mediante la red desarrollada para el proceso de gasificación de la madera de balsa objeto de estudio.

Como se puede observar en la Fig. 4, el ajuste mostrado por la RNA es satisfactorio por lo cual se considera válida para la realización de predicciones sobre el poder calorífico del gas obtenido mediante la gasificación de la madera de balsa sin necesidad de realizar los costosos experimentos necesarios para ello, aunque es válido destacar la necesidad de reentrenamiento periódico de esta red, incorporando nuevos valores mediante la realización de experimentos pasivos para mejorar progresivamente su ajuste.

\section{Conclusiones}

Como conclusiones del trabajo realizado y las mediciones experimentales obtenidas donde se obtuvieron un número de datos suficientes para la realización del análisis requerido para el desarrollo del modelado y la predicción del poder calórico de del gas en el proceso de gasificación de la madera de balsa y la definición de los parámetros de entradas en este proceso.
La aplicación de las herramientas modernas de análisis y modelado implementadas en Matlab permitieron el desarrollo de un modelo de red neuronal artificial de tipo feed-forward con una capa oculta de seis neuronas, a las que se les identificaron los coeficientes bias $(b)$ y los coeficientes de pesos de los nodos $(w)$ mediante el entrenamiento con el $70 \%$ de los datos de entada para la realización de predicciones $P C$ de gas en gasificación de materia orgánica lignocelulósica (biomasa) estudiada esta investigación.

La red neuronal artificial obtenida en este estudio, permite la predicción del poder calorífico resultante de la gasificación de la madera de balsa con un error de 2.6 y un ajuste del $86 \%$ aproximadamente, lo cual ayuda a realizar una adecuada predicción sobre el valor de esta propiedad energética, como alternativa a la carencia de procedimientos analíticos de alta precisión para su determinación.

\section{Referencias}

[1] International Energy Agency (IEA), Good practice guidelines: bioenergy project development and biomass supply, OECD/IEA, 2007, Francia.

[2] P. Basu, Biomass gasification and pyrolysis: practical design and theory, MA, U.S., Elsevier, 2010.

[3] P. Basu, Combustion and gasification in fluidized beds", London: Taylor \& Francis Group/CRC Press, 2006.

[4] A. K. Sharma, "Experimental investigations on a $20 \mathrm{kWe}$, solid biomass gasification system," Biomass Bioenergy, 2011, vol. 35, pp. 421-8.

[5] S. Shabbar, "Thermodynamic equilibrium analysis of coal gasification using Gibbs energy minimization 
method," Energy Convers Manage, 2013, vol. 65, pp. 755-63.

[6] N.S. Barman, "Gasification of biomass in a fixed bed downdraft gasifier: a realistic model including tar," Bioresour Technol., 2012, vol. 107, pp. 505-11.

[7] E. Azzone, "Development of an equilibrium model for the simulation of thermochemical gasification and application to agricultural residues," Renewable Energy, 2012, vol. 46, pp. 248-54.

[8] J. Xie, "Simulation on gasification of forestry residues in fluidized beds by Eulerian Lagrangian approach," Bioresour Technol., 2012, vol. 121, pp. 36-46.

[9] I. Janajreh, M. Al Shrah, "Numerical and experimental investigation of downdraft gasification of woodchips," Energy Convers Manage, 2013, vol. 65, pp. 783-92.

[10] M. P. Arnavat, "Artificial neural network models for biomass gasification in fluidized bed gasifiers," Biomass Bioenergy, 2013, vol. 49, pp. 279-89.

[11] J. Han, "Modeling downdraft biomass gasi?cation process by restricting chemical reaction equilibrium with Aspen Plus," Energy Conversion and Management, 2017, vol. 153, pp. 641-648.

[12] M. Puig-Arnavat, "Review and analysis of biomass gasification models," Renewable and Sustainable Energy Reviews, 2010, vol. 14, pp. 2841-2851.

[13] D. Baruah, "Renewable and Sustainable Energy Reviews," Renewable and Sustainable Energy Reviews, 2014, vol. 39, pp. 806-815.

[14] M. Puig-Arnavat, "Review and analysis of biomass gasification models," Renewable and Sustainable Energy Reviews, 2010, vol. 14, pp. 2841-2851.

[15] M. Fani, M. H. Niri, F. Joda, "A simplified dynamic thermokinetic-based model of wood gasification process," Process Integration and Optimization for Sustainability, 2018, vol. 2, núm. 3, pp. 269-279.
[16] Y. Li, L. Yan, B. Yang, W. Gao, M.R. Farahani, "Simulation of biomass gasification in a fluidized bed by artificial neural network (ANN)," Energy Sources, Part A: Recovery, Utilization, and Environmental Effects, vol. 40, núm. 5, pp. 544-548.

[17] T. Y. Ahmed, "Renewable and Sustainable Energy Reviews", Renewable and Sustainable Energy Reviews, 2012, vol. 16, pp. 2304-2315.

[18] C. Pérez, Redes neuronales a través de ejemplos: aplicaciones con MATLAB, España, 2017.

[19] P. Ponce, Inteligencia artificial con aplicaciones a la ingeniería, España, 2011.

[20] F. Berzal, Redes Neuronales \& Deep Learning, Granada, España, 2018.

[21] C.A. Carvajal-Jara, P. M. Tafur-Escanta,A.H. VillavicencioPoveda, E. R. Gutiérrez-Gualotuña, "Caracterización del poder calorífico de la biomasa residual de cacao CCN51 mediante procesos de gasificación anaeróbico y termoquímico," Cientifica, vol. 22, núm. 2, 2018, pp. 113-123.

[22] E. R. Gutiérrez-Gualotuña, J. A. Soria-Amancha, P. M. Tafur-Escanta, N. Rodríguez-Trujillo, A. H. VillavicencioPoveda, J. Arzola-Ruiz, "Modelos matemáticos de los parámetros energéticos de desempeño de gasificadores tipo downdraft mediante técnicas de regresión," Científica, vol. 23, núm. 1, 2019, pp. 61-81.

[23] M. S. Arroyo-López, F. M. Guerrero-Espinosa, E. R. Gutiérrez-Gualotuña, "Análisis comparativo de la densidad y velocidad de ignición óptimas para la combustión completa del olote perteneciente al Zea Mays L.," Cientifica, vol. 23, núm. 1, 2019, pp. 43-50.

[24] E. R. Gutiérrez Gualotuña, J.Almeida Mera, J. C., J.Arzola Ruiz, "Modelado de indicadores de operación de un gasificador downdraft por redes neuronales para biomasa Eichhornia Crassipes," Ingeniería Energética, vol. 40, núm. 3, 2019, pp. 212-222. 
